\title{
Enhanced translation of leaderless mRNAs under oxidative stress in Escherichia coli
}

41 Programa de Biología Celular y Molecular, ICBM, Facultad de Medicina, Universidad 5 de Chile, Santiago, Chile.

62 Facultad de Ciencias, Universidad de Chile, Santiago, Chile

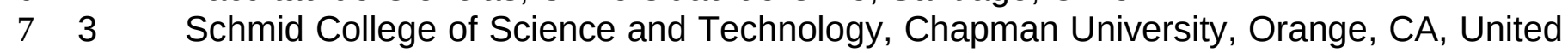
8 States

9 * Correspondence:

10 Assaf Katz

11 askatz@uchile.cl

12 Keywords:

13 ImRNA; Translation initiation; Escherichia coli; Oxidative stress; (p)ppGpp; Translation 14 regulation 


\section{Abstract}

The bacterial response to oxidative stress requires the adaptation of the proteome to

17 the hostile environment. It has been reported that oxidative stress induces a strong and global

18 inhibition of both, transcription and translation. Nevertheless, whereas it is well known that

19 transcription of a small group of genes is induced thanks to transcription factors such as

20 OxyR and SoxR, an equivalent mechanism has not been described for translation. Here we

21 report that whereas canonical translation that depends on Shine Dalgarno recognition is

22 inhibited by oxidative stress in Escherichia coli, the translation of leaderless mRNA (ImRNA)

23 is enhanced under such conditions. Both, inhibition of canonical translation and enhancement

24 of ImRNA translation, depend on the production of (p)ppGpp. We propose that such a

25 mechanism would allow bacteria to rapidly adapt their proteome to hostile conditions and is,

26 perhaps, a general strategy to confront strong stressful conditions.

\section{7}

\section{Significance statement}

The regulation of translation (the production of proteins based on genetic information) is central for the adaptation to environmental changes. In Escherichia coli translation may begin through two alternative pathways. 1.- A canonical initiation that is well understood and is regulated mostly by changes in the accessibility of ribosomes to specific sequences and 2.Initiation of leaderless mRNAs (ImRNAs) that lack these sequences and for which we do not understand the regulation process. Our results indicate that under oxidative stress, the production of (p)ppGpp in E. coli inhibits canonical translation and simultaneously enhances translation of ImRNAs, showing for the first time a natural condition where ImRNA translation is regulated and a role for ( $p) p p G p p$ in this process.

\section{Introduction}

Bacteria are exposed to continual environmental changes, where modification of the proteome allows adaptation to the new conditions. Regulation of bacterial translation is key to this process (Rothschild \& Mancinelli, 2001) and usually depends on changes to the initiation step. This is usually the slowest step of translation, and thus, changes to it will produce the greatest effects on protein production (Gualerzi \& Pon, 2015; Laursen et al., 2005). According to the canonical model, initiation of translation in bacteria starts with the positioning of the 
small ribosomal subunit (30S) and initiator tRNA (fMet-tRNA) at the start codon in the mRNA, in conjunction with initiator factors 1 (IF1), 2 (IF2) and 3 (IF3), generating a pre-initiation complex (Gualerzi \& Pon, 2015; Schmeing \& Ramakrishnan, 2009). The region located upstream of the initiation codon, the leader region, guides the positioning of the start codon in the peptidyl-tRNA site (P-site) of the $30 \mathrm{~S}$ subunit, by the interaction between the Shine Dalgarno (SD) sequence in the mRNA and a highly conserved segment of the 3' end of 16S rRNA called anti-SD (aSD) sequence. In addition, low folding stability (Saito et al., 2020) and interactions of the ribosomal protein S1 and enhancer sequences such as a A/U-rich region upstream of SD may aid binding and positioning of the mRNA to the small ribosomal subunit (Kaminishi et al., 2007; Komarova et al., 2002). Positioning of the $30 \mathrm{~S}$ at the ribosome binding site (RBS) allows binding of the large ribosomal subunit (50S), hydrolysis of GTP by IF2 and the release of initiation factors. This enables the entry of the elongation factor Tu (EF-Tu) with aminoacylated tRNAs and the start of polypeptide synthesis (Gualerzi \& Pon, 2015; Schmeing \& Ramakrishnan, 2009). Given the relevance of the positioning of the 30 S subunit on the RBS, any change of its accessibility will alter the speed of translation initiation. Thus, changes of RNA folding or binding of SRNA and proteins have been observed to be usually involved in translation regulation (Duval et al., 2015).

In addition to the canonical initiation of translation, bacteria may initiate translation from leaderless mRNA (ImRNA). These mRNA are characterized by its lack of the usual 5' UTR.

63 They may present none or a small number of nucleotides and, most importantly, they completely lack any SD sequence. In contrast to the canonical initiation of bacterial translation, ImRNA translation has been proposed to start by binding of $70 \mathrm{~S}$ or smaller $61 \mathrm{~S}$ particles, not 30S (Beck et al., 2016; O'Donnell \& Janssen, 2002; Udagawa et al., 2004). These kind of mRNA without a canonical RBS have been found in phages (e.g., $\lambda$ and P2),

68 transposons (e.g., Tn1721), and several bacterial species (e.g., Mycobacterium smegmatis 69 and Streptomyces clavuligerus) (Cortes et al., 2013; de Groot et al., 2014; Hwang et al., 2019; T. G. Nguyen et al., 2020; Resch et al., 1995). Although they are common in nature,

71 studies targeted to describe transcription initiation sites have usually found only a small 72 number of ImRNA in enterobacteria. For instance, only 20 to 30 ImRNA were found in E. coli 73 K-12 and Salmonella enterica serovar Typhimurium SL1344 (Kröger et al., 2012; Mendoza74 Vargas et al., 2009). Nevertheless, it has been reported in other enterobacteria that 75 transcription from alternative promoters may allow the production of ImRNA under hostile 
76 conditions. For example, an alternative promoter has been described for virF, a transcriptional

77 factor that regulates virulence of Shigella spp. during mammalian cell infection. This

78 alternative promoter generates a ImRNA that allows the production of a functional fragment of

79 the virF regulator (Di Martino et al., 2016), suggesting a possible involvement of ImRNAs

80 translation in the adaptation to host-pathogen conditions.

\section{Translation under oxidative stress}

An example of the hostile conditions commonly confronted by bacteria is oxidative stress. Bacteria are often exposed to various environmental and anthropogenic factors that favor the production of oxidative molecules, which in some conditions, can produce oxidative stress (Imlay, 2019; Kataria \& Ruhal, 2014). One of such conditions is the interaction with human immune cells, such as macrophages and polymorphonuclear leukocytes (G. T. Nguyen et al., 2017; Slauch, 2011). As a consequence, microorganisms have developed diverse strategies to overcome this stress. Whereas the transcriptional regulation of this response has been described in great detail (Chiang \& Schellhorn, 2012; Imlay, 2013), the literature on changes in the translational machinery under oxidative stress is more limited.

91 However, many authors have described a global inhibition of translation as a consequence of 92 oxidative stress (Fasnacht \& Polacek, 2021; Kojima et al., 2007; Nishiyama et al., 2004;

93 Shenton et al., 2006; Zhong et al., 2015; M. Zhu \& Dai, 2019). In addition to this global 94 repression of translation, we and other authors have described important alterations in 95 translation elongation. For instance, an increased error rate in the incorporation of some amino acids (Ling \& Soll, 2010; Steiner et al., 2019; Wu et al., 2014) or decreased elongation rates during translation have been observed (Leiva et al., 2020; Zhong et al., 2015; M. Zhu \& Dai, 2019). The relative contribution of each of these effects is not completely understood. Some groups have proposed that tRNA degradation limits translation elongation, determining

100 the global translation efficiency (Zhong et al., 2015; M. Zhu \& Dai, 2019). Nevertheless, this 101 effect depends on the stressor concentration and is probably affected by other aspects, such 102 as culture conditions and particular strains used. In effect, we have not observed this tRNA 103 degradation using another strain of E. coli (K-12 MG1655). When using $250 \mu \mathrm{M}$ of paraquat 104 to induce oxidative stress, the decreased translation seems to derive mainly from steps 105 different from elongation, probably translation initiation (Leiva et al., 2020). 
107 investigated the impact of this stress on translation initiation. Our results show that while

108 translation of canonical mRNA is inhibited under oxidative stress, that of ImRNAs is strongly

109 induced. Furthermore, the results strongly suggest that these changes depend in part on the 110 production of ( $\mathrm{p})$ ppGpp, an alarmone known to modulate the transcription and translation 111 machinery in response to hostile environmental conditions.

\section{Results}

113 Oxidative stress induces the formation of a ribosomal particle of lower sedimentation coefficient

In order to get some insights about the effects of paraquat on translation, we analyzed 116 the sedimentation coefficient of ribosomes from E. coli cultured in M9r media in the presence 117 of $250 \mu \mathrm{M}$ paraquat. This is a technique commonly used to analyze translation, as it indicates 118 the proportion of ribosomes that are involved in more efficient (polysome fraction) and more 119 inefficient (monosome/70S fraction) translation. Previous assays have shown that under the 120 oxidative stress condition used in this experiment, bacterial replication and protein synthesis 121 are greatly decreased (Leiva et al., 2020). Also, for this strain, the decreased translation 122 produced by this lower concentration of paraquat seems to depend mainly on steps different 123 from elongation (Leiva et al., 2020). To this end, a sedimentation analysis of the components 124 of the translational machinery was carried by ultracentrifugation in sucrose gradients (10125 40\%) (Qin \& Fredrick, 2013). As expected, free 30S, and 50 S subunits, a majority peak 126 corresponding to monosomes (70S) and a fraction of polysomes were observed when 127 analyzing cells cultured under control conditions (Figure 1A) (Qin \& Fredrick, 2013). These 128 peaks were also observed in extracts from cultures stressed with $250 \mu \mathrm{M}$ paraquat for 30 129 minutes (Figure 1B). However, we observed the appearance of a particle of intermediate 130 sedimentation coefficient between the $50 \mathrm{~S}$ subunit and complete ribosome (70S) peaks, 131 whose abundance varies over time (Figure 1C). By interpolation, we have estimated that this 132 particle has a sedimentation coefficient of $60 \pm 0.6 \mathrm{~S}$, very similar to particles observed after 133 addition of Kasuganycin to E. coli cultures, especially when ImRNAs are overexpressed. This 134 antibiotic has been proposed to promote the loss of at least 6 ribosomal proteins of the minor 135 subunit (some of them highly relevant for translation, such as S1 and S12) and induce the 136 translation of ImRNA (Delvillani et al., 2011; Kaberdina et al., 2009). Given the relevance that 137 activation of translation of the ImRNA subset of the transcriptome would have in the 
adaptation to stressful conditions, we decided to further investigate the possibility that translation of ImRNA is induced by paraquat dependent oxidative stress.

\section{Translation of leaderless mRNA is enhanced by oxidative stress}

Canonical and ImRNA initiation are the only two mechanisms known to be capable of initiating translation of the first cistron of bacterial transcripts. To compare the efficiency of translation by both mechanisms under control and oxidative stress conditions, we used two genetic reporter plasmids that code for a biscistronic operon coding for two fluorescent proteins, GFP upstream of mCherry (Figure 2A). One of such plasmids, plasmid pSD, was used to study canonical initiation. In pSD, both reporter genes (gfp and mCherry) have a canonical RBS. A second plasmid, plmRNA, was used to study ImRNA initiation. In plmRNA the first cistron ( $g f p$ ) lacks a leader region, but the second cistron (mCherry) preserves a canonical RBS. Transcription initiation sites for both reporter plasmids were confirmed by reverse transcription coupled to template exchange (Langevin et al., 2013; Y. Y. Zhu et al., 2001). These experiments indicated that the ImRNA transcript presents 3 nucleotides (ATA) upstream of the AUG initiation codon confirming its leaderless nature (Supplementary Table S1). Thus, in both plasmids mCherry initiation operates through the same canonical mechanism, but while initiation of gfp translation depends on canonical mechanisms for pSD, in plmRNA gfp translation may only start through leaderless translation. As both genes, gfp and mCherry, are coded in the same RNA, alterations in the ratio of fluorescence measured from both proteins (GFP/mCherry) will reflect relative changes in the efficiency of both translation mechanisms.

When comparing fluorescence intensity kinetics of both reporters, we observed that translational levels of GFP and mCherry encoded in PSD decrease drastically under oxidative stress (Figure 2BC). In contrast, synthesis of GFP encoded in the leaderless transcript increased under oxidative stress (Figure 2B), while the levels of mCherry (that remains being translated by the canonical mechanism) show a small decrease (Figure $2 \mathrm{C}$ ). Together, these results indicate that a strong inhibition of canonical initiation of translation occurs under oxidative stress, while efficiency of the translation of ImRNA increases with respect to control condition. As a result, we concluded that gfp in an ImRNA context is translated more efficiently than in a canonical context under oxidative stress. This interpretation derives from comparing the ratio between the speed of GFP and mCherry production (Figure $2 \mathrm{E}$ ). As a plot 
169 of the ratio between the values of two straight lines gives an asymptote that tends to the ratio

170 of the slopes of both lines, a similar trend was observed by analyzing the ratio between the

171 levels of GFP and mCherry after 2 hrs of induction (Figure 2DF) or a ratio between the areas

172 under the curves in the linear region of slope $\sim 0$ of a GFP/mCherry vs time plot (Figure 2DG).

173 All these approaches indicated an estimated 10 to 20 fold increase for the efficiency of

174 translation of leaderless reporter with respect to the canonical reporter under oxidative stress

175 induced by $250 \mu \mathrm{M}$ paraquat. pSD reporter does not show relevant changes of the

176 GFP/mCherry ratio because translation efficiency is altered to a similar extent for both gfp and

177 mCherry when it depends on canonical initiation. As the three methods give similar

178 conclusions, further results are only expressed as the ratio of fluorescence at 2 hrs. To

179 confirm this result does not derive from partial degradation of the reporter mRNAs, qRT-PCR

180 was performed with probes targeted to 3 regions of the bicistronic reporter. As shown in

181 supplementary figure S1, there are small changes on the levels of each region as a

182 consequence of oxidative stress. Under oxidative stress, these changes of mRNA levels

183 would produce a small increase of gfp mRNA for the SD reporter and a small increase of 184 mCherry mRNA for the ImRNA reporter. Thus, changes of GFP/mCherry fluorescence does 185 not derive form partial mRNA degradation. If anything, this result suggests that activation of 186 ImRNA translation is stronger than interpreted exclusively from fluorescence changes. To 187 further confirm that the GFP/mCherry method is useful to study alterations in ImRNA 188 translation, we repeated this experiment inducing stress with kasugamycin, an antibiotic that 189 is known to induce ImRNA translation (Kaberdina et al., 2009; Schluenzen et al., 2006; 190 Schuwirth et al., 2006). Kasugamycin induced a similar increase in the GFP/mCherry 191 fluorescence ratio for the plmRNA reporter (Supplementary Figure S2) as observed with 192 paraquat (Figure 2), further supporting our conclusion. Thus, in toto, our results strongly 193 suggest that $250 \mu \mathrm{M}$ paraquat induce the translation of ImRNAs. To our knowledge, this is the 194 first report of increased ImRNA translation in conditions that do not derive from antibiotics 195 stress (Kaberdina et al., 2009) or artificial deletion (Landwehr et al., 2021) or overexpression 196 of genes (Vesper et al., 2011). 
200 (70S) than for the minor subunit (30S) (O'Donnell \& Janssen, 2002), today there is no clarity 201 on the mechanism responsible for initiation of their translation. The formation of specialized 202 ribosomes, responsible for the preferential translation of mRNA under certain stress 203 conditions has been proposed. This process would be mediated by the MazEF toxin-antitoxin 204 system, which would be responsible for partially digesting the 3 ' end of the 16S rRNA of the 205 minor ribosome subunit (30S) and/or complete ribosomes (70S). This mechanism would 206 generate ribosomes lacking 43 nucleotides $\left(70 S^{\Delta 43}\right)$ that contain the aSD sequence, causing a 207 reduction in the affinity for mRNAs with a leader region, but maintaining translation of 208 leaderless transcripts (Temmel et al., 2016; Vesper et al., 2011).

209 To evaluate whether the translation changes observed for the plmRNA reporter under 210 oxidative stress depend on the action of MazEF, a mutant of the mazEF operon was 211 constructed by homologous recombination with PCR products (Datsenko \& Wanner, 2000). 212 From the analysis of the translational kinetics using wild type E. coli K-12 MG1655 (K12) and 213 mutant $E$. coli K-12 MG1655 $\triangle$ mazEF::FRT ( $\triangle$ mazEF), we observed that deletion of the 214 MazEF toxin-antitoxin system does not prevent the strong increase in translation of gfp from 215 the pImRNA reporter under oxidative stress (Figure 3A).

216 To further verify whether MazF or another endoribonuclease are processing the 16S 217 rRNA under oxidative stress induced by paraquat, the integrity of its 3' end was analyzed by 218 real-time RT-PCR. To this end, primers that hybridize at both sides of the proposed ACA 219 cleavage site and indicate the levels of the non-cleaved 16S rRNA were used (primers S7 to 220 Y12 in Figure 3B) (Vesper et al., 2011). A second pair of primers (S7 and X15) hybridizing a 221 region conserved in all 16S rRNA species independent of their processing were used as an 222 internal control of $16 \mathrm{~S}$ rRNA levels. When analyzing the wild type strain, no significant 223 changes were observed in the relative levels of the complete 3 ' end of the 16S rRNA after 224 induction of oxidative stress with $250 \mu \mathrm{M}$ of paraquat. This suggests the absence of digestion 225 at the 3' end of the 16S rRNA under oxidative stress (Figure 3C). Confirming these results, we 226 were unable to detect the expected 43 nucleotide fragment by Northern blot analysis using 227 the same probes that have been previously reported (Vesper et al., 2011) (data not shown). Although in contradiction to the MazF model for ImRNA translation activation, these 230 endoribonuclease would not generate $70 S^{\Delta 43}$ ribosomes. Instead, these researchers found 231 that activation of MazF non-selectively degrades immature rRNA and mRNA (Culviner \& 
232 Laub, 2018; Mets et al., 2017). In accordance with those reports, when analyzing the levels of

233 the 16S rRNA precursor using a primer that hybridizes to a region of the immature rRNA 234 (downstream of the mature 3' extreme, Figure 3B), we observed that the levels of 16S pre235 rRNA decrease dramatically after the induction of oxidative stress (Figure 3D). All together, 236 these results suggest that in accordance with what was published by Mets et al., 2017 and 237 Culviner \& Laub, 2018, in E. coli K-12 MG1655, 70S ${ }^{\Delta 43}$ ribosomes are not generated under 238 oxidative stress. The results are also congruent with a decreased abundance of ribosomal 239 RNA precursors that may derive from either their degradation or decreased transcription.

240 Oxidative stress increases the translational efficiency of ImRNAs naturally found in $E$. 241 coli

242 Our results strongly supports that translation of ImRNA is activated under oxidative 243 stress (Figure 2) in a mechanism that probably involves some changes in the composition of 244 the ribosomal particles (Figure 1), but is independent of the processing of the 16S rRNA 3' 245 extreme (Figure 3). Nevertheless, all these analyzes depend on experiments carried out using 246 the plasmid plmRNA, which generates an artificial leaderless mRNA encoding the 247 heterologous genes gfp and mCherry. However, leaderless transcripts can also be found in 248 nature. For example, as a product of alternative promoters in regulatory genes from phages $\lambda$ 249 and P2, transposon Tn1721, or virF in Shigella spp. (Cortes et al., 2013; Di Martino et al., 250 2016; Resch et al., 1995). The first approaches to understand the mechanism of leaderless 251 translation were carried out with the $c /$ gene of phage $\lambda$. This gene has two described 252 promoters, $P_{R E}$ (promoter for repressor establishment) and $P_{R M}$ (promoter for repressor 253 maintenance). Transcription from $P_{\mathrm{RE}}$ generates a transcript with a $5^{\prime}$ untranslated region of 254403 nucleotides, while transcription beginning at $P_{\mathrm{RM}}$ produces a leaderless mRNA, whose 5' 255 end corresponds to the AUG start codon (Schmeissner et al., 1980; Walz et al., 1976). To 256 evaluate changes in the translation efficiency of the leaderless $\mathrm{cl}$ under oxidative stress, a 257 translation fusion of the first $100 \mathrm{cl}$ codons (from $\mathrm{P}_{\mathrm{RM}}$ ) to gfp was constructed in the vector 258 plmRNA, forming reporter cl-gfp (Figure 4A). As expected, we observed an increase in 259 GFP/mCherry ratio under oxidative stress (Figure 4C). Although activation of ImRNA 260 translation is clear, this enhancement is more discreet than what we have observed using the 261 plmRNA reporter (Figure 2). We are currently unable to explain this difference, but it is 262 possible that it derives from differences between the sequence of the first codons of both 
263 reporters of ImRNA translation, as previous reports have shown idiosyncratic effects of 264 diverse ImRNA reporter sequences (Beck et al., 2016; Shell et al., 2015; Udagawa et al., 265 2004).

266 In addition to the synthesis of ImRNA by transcription from promoters located near to 267 the initiation codon, some authors have proposed that ImRNAs may be also generated 268 through cleavage by MazF of canonical mRNAs (Vesper et al., 2011). Possible MazF targets 269 with their respective ACA cleavage sites have been described in $E$. coli strains that 270 overexpress mazF (Sauert et al., 2016). Four of these genes belong to the SoxRS and OxyR 271 regulons and thus, are potentially involved in the response to oxidative stress by paraquat: $272 \mathrm{fpr}$, katG, nepl and zwf (Seo et al., 2015). Since the transcription initiation site of katG has 273 been described (Tartaglia et al., 1989), this gene was chosen to study whether MazF may be 274 involved in the production of ImRNAs under oxidative stress. To this end, a translation fusion 275 between the 5' extreme of katG (including the 5' UTR and the first 100 codons of the gene) 276 and the gfp reporter was constructed. Furthermore, a point mutation was generated in the 277 ACA cleavage site replacing it with AGA, to obtain a transcript that is not processable by 278 MazF (Figure 4B). When analyzing gfp translation in the wild type strain, we observed no 279 differences between the katG(ACA)-gfp and katG(AGA)-gfp reporters under control conditions 280 (Figure 4D). However, under oxidative stress induced by $250 \mu \mathrm{M}$ of paraquat, we observed a 281 higher GFP/mCherry ratio for the reporter that can be processed by MazF (ACA) (Figure 4D). 282 When repeating these experiments in a strain lacking the toxin-antitoxin system, we did not observe any differences between the reporters, both under control and oxidative stress 284 conditions (Figure 4D). Since we previously observed that MazF does not play a role in the 285 increased translation of ImRNA under oxidative stress (Figure 3A), these latest data suggest 286 that MazF would participate in the processing of the katG transcript, increasing its 287 translational efficiency under oxidative stress. As reporters differ only in the cleavage site 288 described for MazF, it can be proposed that the increased translation of katG(ACA)-gfp is a 289 consequence of the more efficient translation of at least a fraction of the mRNA that is 290 processed into ImRNA. This result is similar to that recently described for the grcA mRNA 291 (MazF target), whose translation increases in a part of the bacterial population when MazF is 292 overexpressed (Nikolic et al., 2017). Nevertheless, care should be taken in considering that 293 induction of ImRNA translation under stress conditions in the WT strain is modest, an effect 294 that may derive from further digestion of transcripts cleaved by MazF (Culviner \& Laub, 2018). 


\section{(p)ppGpp accumulation inhibits translation of canonical mRNAs under oxidative stress}

Our results indicate that under stress conditions, a decrease in ribosomal RNA precursors is observed (Figure 3D). Such decrease may be a consequence of the accumulation of guanosine tetra- or penta-phosphate ((p)ppGpp) (Sanchez-Vazquez et al., 2019). This is a nucleotide alarmone produced when E. coli or other bacteria are exposed to decreased amino acids availability or several other conditions, including oxidative stress (Chang et al., 2002). It has been determined that this molecule is capable of interacting with around 30 proteins involved in different processes, including some involved in ribosome biogenesis and translation. As a consequence, (p)ppGpp inhibits transcription and translation of several genes (Zhang et al., 2018). Although it is well known that transcription of some genes is enhanced by (p)ppGpp (Sanchez-Vazquez et al., 2019), we are unaware of any report regarding its effects on ImRNA translation. To determine whether this metabolite plays a role in the regulation of leaderless translation, we analyzed the efficiency of GFP production from pSD and plmRNA reporters in a mutant lacking the relA and spoT genes $(\Delta r e l A:: F R T$ $\triangle$ spoT::FRT or $\Delta r e l A \Delta s p o T$ ), responsible for the synthesis of (p)ppGpp in $E$. coli (46). As mutants that cannot produce (p)ppGpp are unable to replicate in culture media devoid of most amino acids (Potrykus et al., 2011), we initially tried to perform the experiments in M9 media 312 supplemented with triptone, a casein digest that contains all amino acids. Unexpectedly, in 313 this culture medium we did not observe ImRNA translation in either the WT or the $\Delta$ relA $\triangle$ spoT 314 strains, both under control or oxidative stress conditions (Data not shown). To overcome this 315 limitation, WT and $\Delta r e I A \Delta s p o T$ strains were cultured in M9 media supplemented with tryptone.

316 Before performing the experiment, cultures were cleaned in M9 media and resuspended in 317 M9 media with branched amino acids where they were used to test the production of GFP 318 and mCherry. Using this protocol, we observed that ImRNA translation is activated in the WT 319 strain under oxidative stress, replicating our previous observations. Nevertheless, the $320 \Delta$ relA $\triangle$ spoT strain was unable to translate the ImRNA gfp reporter under any conditions. If we 321 instead resuspended the cell in M9 media containing tryptone, the ImRNA reporter was not 322 translated in any of the conditions or strains (Figure 5). Interestingly, deletion of the genes 323 involved in (p)ppGpp synthesis also prevented the inhibition of canonical GFP and mCherry 324 translation under oxidative stress (Figure 5). Altogether, these results show that ImRNA 325 cannot be translated in strains impeded to produce sufficient levels of (p)ppGpp either due to 
mutation of relA and spoT or because of a high amino acids availability. Thus, our work strongly suggests that in addition to inhibiting canonical translation, (p)ppGpp is required for the activation of ImRNA translation at least under oxidative stress. Deletion of only relA was not enough to prevent ImRNA translation under oxidative stress, although it did affect translation of canonical mRNAs (Supplementary Figure S3).

Part of the (p)ppGpp response is mediated by Lon protease activation that leads to degradation of ribosomal proteins (Kuroda, 2001). However, lon deletion does not prevent gfp expression from the ImRNA reporter (Supplementary Figure S3) indicating that ImRNA translation does not require the Lon dependent degradation of ribosomal proteins.

\section{Discussion}

Because initiation is usually the slowest step of translation, regulation of mRNA translation usually depends on alterations of this step. This is usually achieved by changes to the accessibility of the RBS, ultimately controlling binding of the 30 S subunit (Duval et al., 2015). In addition to this regulation of translation of particular genes, global inhibition of translation has been observed as a response to several hostile conditions such as the stationary phase of bacterial cultures (Kato et al., 2010), when $\mathrm{Mg}^{+2}$ is limiting (Pontes et al., 2016) or as a response to cold shock (Vila-Sanjurjo et al., 2004). This decrease in translation usually depends on the inhibition of ribosomes, but in some cases changes in the accessibility of the RBS also plays a role. One of the best described mechanisms for global inhibition of bacterial translation is the early response to cold shock in E. coli. As temperature decreases, mRNA folds, preventing access of the ribosome to correct translation initiation sites. Thus, translation must be globally repressed, preventing initiation at incorrect sites. As a response, most $E$. coli ribosomes enter a hibernation state induced by binding of YfiA protein to the $P$ and A sites of complete ribosomes (70S). This YfiA-70S complex is unable to catalyze translation (Vila-Sanjurjo et al., 2004). Nevertheless, in order to confront the cold shock, it is not enough to block mis-translation of folded mRNA. Bacteria must additionally enhance translation of genes that may aid its ability to overcome the stress conditions. It has been observed that the reduced number of functional ribosomes selectively translate a minor subset of the available mRNAs that code for RNA chaperons and other proteins that allow a 
355

better adaptation to cold conditions. All these mRNA fold under cold conditions to a structure where the RBS and enhancer sequences remain exposed to binding by the $30 \mathrm{~S}$ allowing translation initiation (Vila-Sanjurjo et al., 2004). Thus, the early cold shock response is characterized by a global inhibition of translation together with the induction of translation of a subset of genes required for cold shock response.

The response to oxidative stress seems to follow a similar behavior to what has been observed under cold shock, although mechanisms appear to be different. Several authors have observed a global inhibition of translation (Kojima et al., 2007; Nishiyama et al., 2004; Shenton et al., 2006; Zhong et al., 2015; M. Zhu \& Dai, 2019). Some of them have indicated that this inhibition would derive from massive tRNA degradation (Zhong et al., 2015; M. Zhu \& Dai, 2019). Nevertheless, it seems that this would be a strain dependent behavior, as strain K-12 MG1655 used for this report does not show such massive decrease in tRNA content (Leiva et al., 2020). Here we have observed that (p)ppGpp production may be responsible for at least part of such inhibition. Perhaps, this may derive from the ability of (p)ppGpp to bind to some translation factors using the GTP binding sites (Kanjee et al., 2012). Interestingly, this is the case of IF2 (Mitkevich et al., 2010) that is required for canonical, but not for ImRNA translation (Udagawa et al., 2004). Additional inhibition may derive from direct oxidation of proteins and RNA involved in the translation process (Katz \& Orellana, 2012; Liu et al., 2012; Willi et al., 2018). Independent of the mechanism underlying global translation inhibition, bacteria will need to preferentially translate genes that allow adaptation to oxidative conditions. Otherwise, the effects of transcriptional control on bacterial adaptation would be limited by a slow translation apparatus. Here we have observed that translation of ImRNA is activated under oxidative stress conditions, apparently by the same ( $p$ )ppGpp that inhibits canonical translation. This activation may be an indirect consequence of the increased availability of ribosomes that show decreased translation of canonical mRNAs when translation is inhibited by ( $p$ )ppGpp. Also, high levels of aminoacylated initiator tRNA, which probably increase as a consequence of canonical translation inhibition, have been shown to enhance ImRNA translation in vitro and could also be involved in the regulatory process (Udagawa et al., 2004). Recently, the ATPase YchF of E. coli has been shown to bind the ribosomes and apparently inhibit ImRNA translation (Landwehr et al., 2021). Thus, it is possible that (p)ppGpp may inhibit YchF, although it is also possible that YchF somehow prevents ( $p$ )ppGpp production or that it controls ImRNA translation by a ( $p$ )ppGpp 
independent mechanism.

Understanding the mechanisms involved in (p)ppGpp activation of ImRNA translation will require further investigation. Nevertheless, the apparent ability of (p)ppGpp to simultaneously inhibit canonical translation and enhance ImRNA translation could allow for the selective translation of a subset of the transcriptome under oxidative stress. Additional experiments will be also required to determine this subset of ImRNAs, allowing a better understanding of the translational control for the adaptation to oxidative stress. If confirmed, these results suggest that bacteria may confront diverse hostile conditions through a common strategy of translation regulation, that is, a global inhibition of translation together with a concomitant activation of the translation of particular genes.

\section{Materials and Methods}

A brief description of the main methods used in this work is provided here. A detailed description can be found at the supplementary methods section.

\section{Strains and culture media}

All experiments performed in this work used wild-type (WT) E. coli K-12 MG1655 strain. Strains were cultured in either lysogeny broth (LB) media (1\% tryptone, 0.5\% yeast

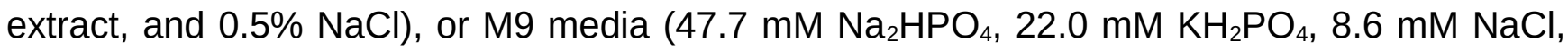
18.7 $\mathrm{mM} \mathrm{NH}_{4} \mathrm{Cl}, 2 \mathrm{mM} \mathrm{MgSO}_{4}, 0.1 \mathrm{mM} \mathrm{CaCl}_{2}$, and 0.4\% Glycerol). When indicated, branched amino acids (isoleucine, leucine, and valine $50 \mu \mathrm{g} / \mathrm{ml}$ each), 0.1\% tryptone, 0.4\% arabinose $0.4 \%$, ampicillin $(100 \mu \mathrm{g} / \mathrm{ml})$ or $250 \mu \mathrm{M}$ paraquat were added to the culture media.

\section{Analysis of polysomes}

The detailed protocol for analysis of polysomes has been described by Qin and Kurt (2013). In brief, $50 \mathrm{ml}$ of M9 media supplemented with branched amino acids were inoculated with saturated overnight culture in M9 media supplemented with tryptone and grown at $37^{\circ} \mathrm{C}$ in an orbital shaker. When indicated, the media also contained $250 \mu \mathrm{M}$ paraquat. When bacteria reached mid-log phase (OD 600 -0.4-0.6), cells were collected, lized and prepared for ultracentrifugation as indicated in the supplementary methods. Samples were then loaded 
416 on a 10\%-40\% sucrose gradient and centrifuged in a SW41 rotor at 35000 RPM for 3 hours.

417 Finally, samples were analyzed using an in-line UV detector (ISCO/Brandel system).

\section{Translation Efficiency Analyses}

$50 \mu \mathrm{l}$ aliquot of mid-log phase cultures were diluted in a 96-well optical-bottom plate with $150 \mu \mathrm{l}$ fresh M9 media supplemented with branched amino acids (50 $\mu \mathrm{g} / \mathrm{ml}$ each) and 421 arabinose (0.4\% final concentration). When indicated, culture media contained paraquat (250 $422 \mu \mathrm{M}$ final concentration). Also 0,5\% tryptone was used instead of branched amino acids in 423 some experiments. Plates were further shaken at $37^{\circ} \mathrm{C}$. OD 600 and fluorescence intensity of 424 GFP (Ex. $480 \pm 4.5$ nm, Em. $515 \pm 10$ nm) and mCherry (Ex. $555 \pm 4.5$ nm, Em. $600 \pm 10$ nm) 425 were measured in a microplate reader (Infinite M200 PRO, Tecan). In all experiments, strains 426 transformed with pBAD30 (parental plasmid of pBAD30SFIT) were used to subtract the 427 fluorescence background. A detailed description of the protocol can be found at the 428 supplementary methods section.

\section{Author contribution}

The project was originally designed by $\mathrm{MI}, \mathrm{AK}$ and $\mathrm{OO}$. All experiments were 431 performed by LL. AK, MI and OO supervised experiments and data interpretation. LL and AK 432 wrote the paper with contributions from all authors. All authors reviewed and approved the 433 final version of the manuscript.

\section{Funding}

435 This work was supported by Vicerrectoría de Investigación y Desarrollo (VID), 436 Universidad de Chile [ENL05/18 to A.K]; Fondo Nacional de Desarrollo Científico y 437 Tecnológico [1191074 to A.K. and 1190552 to O.O]; National Institutes of Health Grant 438 [GM65183 to M.I.]; and a CONICYT Doctorado Nacional scholarship from Comisión Nacional 439 de Investigación Científica y Tecnológica [21151441 to L.L].

\section{References}

441 Beck, H. J., Fleming, I. M. C., \& Janssen, G. R. (2016). 5'-Terminal AUGs in Escherichia 442 coli mRNAs with Shine-Dalgarno Sequences: Identification and Analysis of Their Roles in 
Non-Canonical Translation Initiation.

PLOS

ONE,

11(7)

e0160144. https://doi.org/10.1371/journal.pone.0160144

\section{5}

Chang, D.-E., Smalley, D. J., \& Conway, T. (2002). Gene expression profiling of Escherichia coli growth transitions: An expanded stringent response model. Molecular Microbiology, 45(2), 289-306. https://doi.org/10.1046/j.1365-2958.2002.03001.x

Chiang, S. M., \& Schellhorn, H. E. (2012). Regulators of oxidative stress response genes in Escherichia coli and their functional conservation in bacteria. Archives of Biochemistry and Biophysics, 525(2), 161-169. https://doi.org/10.1016/j.abb.2012.02.007

Cortes, T., Schubert, O. T., Rose, G., Arnvig, K. B., Comas, I., Aebersold, R., \& Young, D. B. (2013). Genome-wide Mapping of Transcriptional Start Sites Defines an Extensive Leaderless Transcriptome in Mycobacterium tuberculosis. Cell Reports, 5(4), 1121-1131. https://doi.org/10.1016/j.celrep.2013.10.031

Culviner, P. H., \& Laub, M. T. (2018). Global Analysis of the E. coli Toxin MazF Reveals Widespread Cleavage of mRNA and the Inhibition of rRNA Maturation and Ribosome Biogenesis. Molecular Cell, 70(5), 868-880.e10. https://doi.org/10.1016/j.molcel.2018.04.026

Datsenko, K. A., \& Wanner, B. L. (2000). One-step inactivation of chromosomal genes in Escherichia coli K-12 using PCR products. Proceedings of the National Academy of Sciences, 97(12), 6640-6645. https://doi.org/10.1073/pnas.120163297

de Groot, A., Roche, D., Fernandez, B., Ludanyi, M., Cruveiller, S., Pignol, D., Vallenet, D., Armengaud, J., \& Blanchard, L. (2014). RNA Sequencing and Proteogenomics Reveal the Importance of Leaderless mRNAs in the Radiation-Tolerant Bacterium Deinococcus deserti. Genome Biology and Evolution, 6(4), 932-948. https://doi.org/10.1093/gbe/evu069

Delvillani, F., Papiani, G., Dehò, G., \& Briani, F. (2011). S1 ribosomal protein and the interplay between translation and mRNA decay. Nucleic Acids Research, 39(17), 7702-7715. https://doi.org/10.1093/nar/gkr417

Di Martino, M. L., Romilly, C., Wagner, E. G. H., Colonna, B., \& Prosseda, G. (2016). One Gene and Two Proteins: A Leaderless mRNA Supports the Translation of a Shorter Form of the Shigella VirF Regulator. MBio, 7(6), e01860-16. https://doi.org/10.1128/mBio.01860-16

Duval, M., Simonetti, A., Caldelari, I., \& Marzi, S. (2015). Multiple ways to regulate translation initiation in bacteria: Mechanisms, regulatory circuits, dynamics. Biochimie, 114, 18-29. https://doi.org/10.1016/j.biochi.2015.03.007

Fasnacht, M., \& Polacek, N. (2021). Oxidative Stress in Bacteria and the Central Dogma of Molecular Biology. Frontiers in Molecular Biosciences, 8, 671037. https://doi.org/10.3389/fmolb.2021.671037

Gualerzi, C. O., \& Pon, C. L. (2015). Initiation of mRNA translation in bacteria: Structural and dynamic aspects. Cellular and Molecular Life Sciences, 72(22), 4341-4367. 


\section{https://doi.org/10.1007/s00018-015-2010-3}

Hauryliuk, V., Atkinson, G. C., Murakami, K. S., Tenson, T., \& Gerdes, K. (2015). Recent functional insights into the role of (p)ppGpp in bacterial physiology. Nature Reviews Microbiology, 13(5), 298-309. https://doi.org/10.1038/nrmicro3448

Hwang, S., Lee, N., Jeong, Y., Lee, Y., Kim, W., Cho, S., Palsson, B. O., \& Cho, B.-K. (2019). Primary transcriptome and translatome analysis determines transcriptional and translational regulatory elements encoded in the Streptomyces clavuligerus genome. Nucleic Acids Research, 47(12), 6114-6129. https://doi.org/10.1093/nar/gkz471

Imlay, J. A. (2013). The molecular mechanisms and physiological consequences of oxidative stress: Lessons from a model bacterium. Nature Reviews Microbiology, 11(7), 443454. https://doi.org/10.1038/nrmicro3032

Imlay, J. A. (2019). Where in the world do bacteria experience oxidative stress?: Oxidative stress in natural environments. Environmental Microbiology, 21(2), 521-530. https://doi.org/10.1111/1462-2920.14445

Kaberdina, A. C., Szaflarski, W., Nierhaus, K. H., \& Moll, I. (2009). An Unexpected Type of Ribosomes Induced by Kasugamycin: A Look into Ancestral Times of Protein Synthesis? Molecular Cell, 33(2), 227-236. https://doi.org/10.1016/j.molcel.2008.12.014

Kaminishi, T., Wilson, D. N., Takemoto, C., Harms, J. M., Kawazoe, M., Schluenzen, F., Hanawa-Suetsugu, K., Shirouzu, M., Fucini, P., \& Yokoyama, S. (2007). A Snapshot of the 30S Ribosomal Subunit Capturing mRNA via the Shine-Dalgarno Interaction. Structure, 15(3), 289-297. https://doi.org/10.1016/j.str.2006.12.008

Kanjee, U., Ogata, K., \& Houry, W. A. (2012). Direct binding targets of the stringent response alarmone (p)ppGpp: Protein targets of ppGpp. Molecular Microbiology, 85(6), 10291043. https://doi.org/10.1111/j.1365-2958.2012.08177.x

Kataria, R., \& Ruhal, R. (2014). Microbiological Metabolism Under Chemical Stress. In Microbial Biodegradation and Bioremediation (pp. 497-509). Elsevier. https://doi.org/10.1016/B978-0-12-800021-2.00021-2

Kato, T., Yoshida, H., Miyata, T., Maki, Y., Wada, A., \& Namba, K. (2010). Structure of the $100 S$ Ribosome in the Hibernation Stage Revealed by Electron Cryomicroscopy. Structure, 18(6), 719-724. https://doi.org/10.1016/j.str.2010.02.017

Katz, A., \& Orellana, O. (2012). Protein Synthesis and the Stress Response. In M. Biyani (Ed.), Cell-Free Protein Synthesis. InTech. https://doi.org/10.5772/50311

Kojima, K., Oshita, M., Nanjo, Y., Kasai, K., Tozawa, Y., Hayashi, H., \& Nishiyama, Y. (2007). Oxidation of elongation factor $G$ inhibits the synthesis of the D1 protein of photosystem II. Molecular Microbiology, 65(4), 936-947. https://doi.org/10.1111/j.13652958.2007.05836.x 
Komarova, A. V., Tchufistova, L. S., Supina, E. V., \& Boni, I. V. (2002). Protein S1 counteracts the inhibitory effect of the extended Shine-Dalgarno sequence on translation. RNA, 8(9), 1137-1147. https://doi.org/10.1017/S1355838202029990

Kröger, C., Dillon, S. C., Cameron, A. D. S., Papenfort, K., Sivasankaran, S. K., Hokamp, K., Chao, Y., Sittka, A., Hébrard, M., Händler, K., Colgan, A., Leekitcharoenphon, P., Langridge, G. C., Lohan, A. J., Loftus, B., Lucchini, S., Ussery, D. W., Dorman, C. J., Thomson, N. R., ... Hinton, J. C. D. (2012). The transcriptional landscape and small RNAs of Salmonella enterica serovar Typhimurium. Proceedings of the National Academy of Sciences, 109(20), E1277-E1286. https://doi.org/10.1073/pnas.1201061109

Kuroda, A. (2001). Role of Inorganic Polyphosphate in Promoting Ribosomal Protein Degradation by the Lon Protease in E. coli. Science, 293(5530), 705-708. https://doi.org/10.1126/science.1061315

Landwehr, V., Milanov, M., Angebauer, L., Hong, J., Jüngert, G., Hiersemenzel, A., Siebler, A., Schmit, F., Öztürk, Y., Dannenmaier, S., Drepper, F., Warscheid, B., \& Koch, H.-G. (2021). The Universally Conserved ATPase YchF Regulates Translation of Leaderless mRNA in Response to Stress Conditions. Frontiers in Molecular Biosciences, 8, 643696. https://doi.org/10.3389/fmolb.2021.643696

Langevin, S. A., Bent, Z. W., Solberg, O. D., Curtis, D. J., Lane, P. D., Williams, K. P., Schoeniger, J. S., Sinha, A., Lane, T. W., \& Branda, S. S. (2013). Peregrine: A rapid and unbiased method to produce strand-specific RNA-Seq libraries from small quantities of starting material. RNA Biology, 10(4), 502-515. https://doi.org/10.4161/rna.24284

Laursen, B. S., Sørensen, H. P., Mortensen, K. K., \& Sperling-Petersen, H. U. (2005). Initiation of Protein Synthesis in Bacteria. Microbiology and Molecular Biology Reviews, 69(1), 101-123. https://doi.org/10.1128/MMBR.69.1.101-123.2005

Leiva, L. E., Pincheira, A., Elgamal, S., Kienast, S. D., Bravo, V., Leufken, J., Gutiérrez, D., Leidel, S. A., Ibba, M., \& Katz, A. (2020). Modulation of Escherichia coli Translation by the Specific Inactivation of tRNA ${ }^{\text {Gly }}$ Under Oxidative Stress. Frontiers in Genetics, 11, 856. https://doi.org/10.3389/fgene.2020.00856

Ling, J., \& Soll, D. (2010). Severe oxidative stress induces protein mistranslation through impairment of an aminoacyl-tRNA synthetase editing site. Proceedings of the National Academy of Sciences, 107(9), 4028-4033. https://doi.org/10.1073/pnas.1000315107

Liu, M., Gong, X., Alluri, R. K., Wu, J., Sablo, T., \& Li, Z. (2012). Characterization of RNA damage under oxidative stress in Escherichia coli. Biological Chemistry, 393(3), $123-132$. https://doi.org/10.1515/hsz-2011-0247

Mendoza-Vargas, A., Olvera, L., Olvera, M., Grande, R., Vega-Alvarado, L., Taboada, B., Jimenez-Jacinto, V., Salgado, H., Juárez, K., Contreras-Moreira, B., Huerta, A. M., ColladoVides, J., \& Morett, E. (2009). Genome-Wide Identification of Transcription Start Sites, 
Promoters and Transcription Factor Binding Sites in E. coli. PLoS ONE, 4(10), e7526. https://doi.org/10.1371/journal.pone.0007526

Mets, T., Lippus, M., Schryer, D., Liiv, A., Kasari, V., Paier, A., Maiväli, Ü., Remme, J., Tenson, T., \& Kaldalu, N. (2017). Toxins MazF and MqsR cleave Escherichia coli rRNA precursors at multiple sites. RNA Biology, 14(1), 124-135. https://doi.org/10.1080/15476286.2016.1259784

Mitkevich, V. A., Ermakov, A., Kulikova, A. A., Tankov, S., Shyp, V., Soosaar, A., Tenson, T., Makarov, A. A., Ehrenberg, M., \& Hauryliuk, V. (2010). Thermodynamic Characterization of ppGpp Binding to EF-G or IF2 and of Initiator tRNA Binding to Free IF2 in the Presence of GDP, GTP, or ppGpp. Journal of Molecular Biology, 402(5), 838-846. https://doi.org/10.1016/j.jmb.2010.08.016

Nguyen, G. T., Green, E. R., \& Mecsas, J. (2017). Neutrophils to the ROScue: Mechanisms of NADPH Oxidase Activation and Bacterial Resistance. Frontiers in Cellular and Infection Microbiology, 7, 373. https://doi.org/10.3389/fcimb.2017.00373

Nguyen, T. G., Vargas-Blanco, D. A., Roberts, L. A., \& Shell, S. S. (2020). The Impact of Leadered and Leaderless Gene Structures on Translation Efficiency, Transcript Stability, and Predicted Transcription Rates in Mycobacterium smegmatis. Journal of Bacteriology, 202(9), e00746-19. https://doi.org/10.1128/JB.00746-19

Nikolic, N., Didara, Z., \& Moll, I. (2017). MazF activation promotes translational heterogeneity of the grcA mRNA in Escherichia coli populations. PeerJ, 5, e3830. https://doi.org/10.7717/peerj.3830

Nishiyama, Y., Allakhverdiev, S. I., Yamamoto, H., Hayashi, H., \& Murata, N. (2004). Singlet Oxygen Inhibits the Repair of Photosystem II by Suppressing the Translation Elongation of the D1 Protein in Synechocystis sp. PCC 6803. Biochemistry, 43(35), 1132111330. https://doi.org/10.1021/bi036178q

O'Donnell, S. M., \& Janssen, G. R. (2002). Leaderless mRNAs Bind 70S Ribosomes More Strongly than 30S Ribosomal Subunits in Escherichia coli. Journal of Bacteriology, 184(23), 6730-6733. https://doi.org/10.1128/JB.184.23.6730-6733.2002

Pontes, M. H., Yeom, J., \& Groisman, E. A. (2016). Reducing Ribosome Biosynthesis Promotes Translation during Low Mg 2+ Stress. Molecular Cell, 64(3), 480-492. https://doi.org/10.1016/j.molcel.2016.05.008

Potrykus, K., Murphy, H., Philippe, N., \& Cashel, M. (2011). ppGpp is the major source of growth rate control in E. coli: PpGpp and growth rate control. Environmental Microbiology, 13(3), 563-575. https://doi.org/10.1111/j.1462-2920.2010.02357.x

Qin, D., \& Fredrick, K. (2013). Analysis of Polysomes from Bacteria. In Methods in Enzymology (Vol. 530, pp. 159-172). Elsevier. https://doi.org/10.1016/B978-0-12-420037- 
604

605

606

607

608

609

610

611

612

613

614

615

616

617

618

619

620

621

622

623

\section{$1.00008-7$}

Resch, A., Tedin, K., Graschopf, A., Haggård-Ljungquist, E., \& Bläsi, U. (1995). Ternary complex formation on leaderless phage mRNA. FEMS Microbiology Reviews, 17(1-2), 151157. https://doi.org/10.1111/j.1574-6976.1995.tb00197.x

Rothschild, L. J., \& Mancinelli, R. L. (2001). Life in extreme environments. Nature, 409(6823), 1092-1101. https://doi.org/10.1038/35059215

Saito, K., Green, R., \& Buskirk, A. R. (2020). Translational initiation in E. coli occurs at the correct sites genome-wide in the absence of mRNA-rRNA base-pairing. ELife, 9, e55002. https://doi.org/10.7554/eLife.55002

Sanchez-Vazquez, P., Dewey, C. N., Kitten, N., Ross, W., \& Gourse, R. L. (2019). Genome-wide effects on Escherichia coli transcription from ppGpp binding to its two sites on RNA polymerase. Proceedings of the National Academy of Sciences, 116(17), 8310-8319. https://doi.org/10.1073/pnas.1819682116

Sauert, M., Wolfinger, M. T., Vesper, O., Müller, C., Byrgazov, K., \& Moll, I. (2016). The MazF-regulon: A toolbox for the post-transcriptional stress response in Escherichia coli. Nucleic Acids Research, 44(14), 6660-6675. https://doi.org/10.1093/nar/gkw115

Schluenzen, F., Takemoto, C., Wilson, D. N., Kaminishi, T., Harms, J. M., HanawaSuetsugu, K., Szaflarski, W., Kawazoe, M., Shirouzu, M., Shirouzo, M., Nierhaus, K. H., Yokoyama, S., \& Fucini, P. (2006). The antibiotic kasugamycin mimics mRNA nucleotides to destabilize tRNA binding and inhibit canonical translation initiation. Nature Structural \& Molecular Biology, 13(10), 871-878. https://doi.org/10.1038/nsmb1145

Schmeing, T. M., \& Ramakrishnan, V. (2009). What recent ribosome structures have revealed about the mechanism of translation. Nature, 461(7268), 1234-1242. https://doi.org/10.1038/nature08403

Schmeissner, U., Court, D., Shimatake, H., \& Rosenberg, M. (1980). Promoter for the establishment of repressor synthesis in bacteriophage lambda. Proceedings of the National Academy of Sciences, 77(6), 3191-3195. https://doi.org/10.1073/pnas.77.6.3191

Schuwirth, B. S., Day, J. M., Hau, C. W., Janssen, G. R., Dahlberg, A. E., Cate, J. H. D., \& Vila-Sanjurjo, A. (2006). Structural analysis of kasugamycin inhibition of translation. Nature Structural \& Molecular Biology, 13(10), 879-886. https://doi.org/10.1038/nsmb1150

Seo, S. W., Kim, D., Szubin, R., \& Palsson, B. O. (2015). Genome-wide Reconstruction of OxyR and SoxRS Transcriptional Regulatory Networks under Oxidative Stress in Escherichia coli K-12 MG1655. Cell Reports, 12(8), 1289-1299. https://doi.org/10.1016/j.celrep.2015.07.043

Shell, S. S., Wang, J., Lapierre, P., Mir, M., Chase, M. R., Pyle, M. M., Gawande, R., Ahmad, R., Sarracino, D. A., loerger, T. R., Fortune, S. M., Derbyshire, K. M., Wade, J. T., \& 
624 Gray, T. A. (2015). Leaderless Transcripts and Small Proteins Are Common Features of the

625 Mycobacterial Translational Landscape. PLOS Genetics, 11(11), e1005641.

626 https://doi.org/10.1371/journal.pgen.1005641

627 Shenton, D., Smirnova, J. B., Selley, J. N., Carroll, K., Hubbard, S. J., Pavitt, G. D., Ashe, 628 M. P., \& Grant, C. M. (2006). Global Translational Responses to Oxidative Stress Impact 629 upon Multiple Levels of Protein Synthesis. Journal of Biological Chemistry, 281(39), 29011630 29021. https://doi.org/10.1074/jbc.M601545200

631 Slauch, J. M. (2011). How does the oxidative burst of macrophages kill bacteria? Still an 632 open question: How do phagocytic ROS kill bacteria? Molecular Microbiology, 80(3), 580633 583. https://doi.org/10.1111/j.1365-2958.2011.07612.x

634 Steiner, R. E., Kyle, A. M., \& Ibba, M. (2019). Oxidation of phenylalanyl-tRNA synthetase 635 positively regulates translational quality control. Proceedings of the National Academy of 636 Sciences, 116(20), 10058-10063. https://doi.org/10.1073/pnas.1901634116

637 Tartaglia, L. A., Storz, G., \& Ames, B. N. (1989). Identification and molecular analysis of 638 oxyR-regulated promoters important for the bacterial adaptation to oxidative stress. Journal of Molecular Biology, 210(4), 709-719. https://doi.org/10.1016/0022-2836(89)90104-6

Temmel, H., Müller, C., Sauert, M., Vesper, O., Reiss, A., Popow, J., Martinez, J., \& Moll, I. (2016). The RNA ligase RtcB reverses MazF-induced ribosome heterogeneity in Escherichia coli. Nucleic Acids Research, 45(8), 4708-4721. https://doi.org/10.1093/nar/gkw1018

Udagawa, T., Shimizu, Y., \& Ueda, T. (2004). Evidence for the Translation Initiation of Leaderless mRNAs by the Intact $70 \mathrm{~S}$ Ribosome without Its Dissociation into Subunits in Eubacteria. Journal of Biological Chemistry, 279(10), 8539-8546. https://doi.org/10.1074/jbc.M308784200

Vesper, O., Amitai, S., Belitsky, M., Byrgazov, K., Kaberdina, A. C., Engelberg-Kulka, H., \& Moll, I. (2011). Selective Translation of Leaderless mRNAs by Specialized Ribosomes Generated by MazF in Escherichia coli. Cell, 147(1), 147-157. https://doi.org/10.1016/j.cell.2011.07.047

Vila-Sanjurjo, A., Schuwirth, B.-S., Hau, C. W., \& Cate, J. H. D. (2004). Structural basis for the control of translation initiation during stress. Nature Structural \& Molecular Biology, 11(11), 1054-1059. https://doi.org/10.1038/nsmb850

Walz, A., Pirrotta, V., \& Ineichen, K. (1976). $\lambda$ represser regulates the switch between PR and Prm promoters. Nature, 262(5570), 665-669. https://doi.org/10.1038/262665a0

Willi, J., Küpfer, P., Evéquoz, D., Fernandez, G., Katz, A., Leumann, C., \& Polacek, N. (2018). Oxidative stress damages rRNA inside the ribosome and differentially affects the catalytic center. Nucleic Acids Research, 46(4), 1945-1957.

659 https://doi.org/10.1093/nar/gkx1308 
660 Wu, J., Fan, Y., \& Ling, J. (2014). Mechanism of oxidant-induced mistranslation by 661 threonyl-tRNA synthetase. Nucleic Acids Research, 42(10), 6523-6531. 662 https://doi.org/10.1093/nar/gku271

663 Zhang, Y., Zborníková, E., Rejman, D., \& Gerdes, K. (2018). Novel (p)ppGpp Binding and 664 Metabolizing Proteins of Escherichia coli. MBio, 9(2). https://doi.org/10.1128/mBio.02188-17

665 Zhong, J., Xiao, C., Gu, W., Du, G., Sun, X., He, Q.-Y., \& Zhang, G. (2015). Transfer RNAs 666 Mediate the Rapid Adaptation of Escherichia coli to Oxidative Stress. PLOS Genetics, 11(6), 667 e1005302. https://doi.org/10.1371/journal.pgen.1005302

668 Zhu, M., \& Dai, X. (2019). Maintenance of translational elongation rate underlies the 669 survival of Escherichia coli during oxidative stress. Nucleic Acids Research, 47(14), 7592670 7604. https://doi.org/10.1093/nar/gkz467

671 Zhu, Y. Y., Machleder, E. M., Chenchik, A., Li, R., \& Siebert, P. D. (2001). Reverse 672 transcriptase template switching: A SMART approach for full-length cDNA library 673 construction. BioTechniques, 30(4), 892-897. https://doi.org/10.2144/01304pf02 


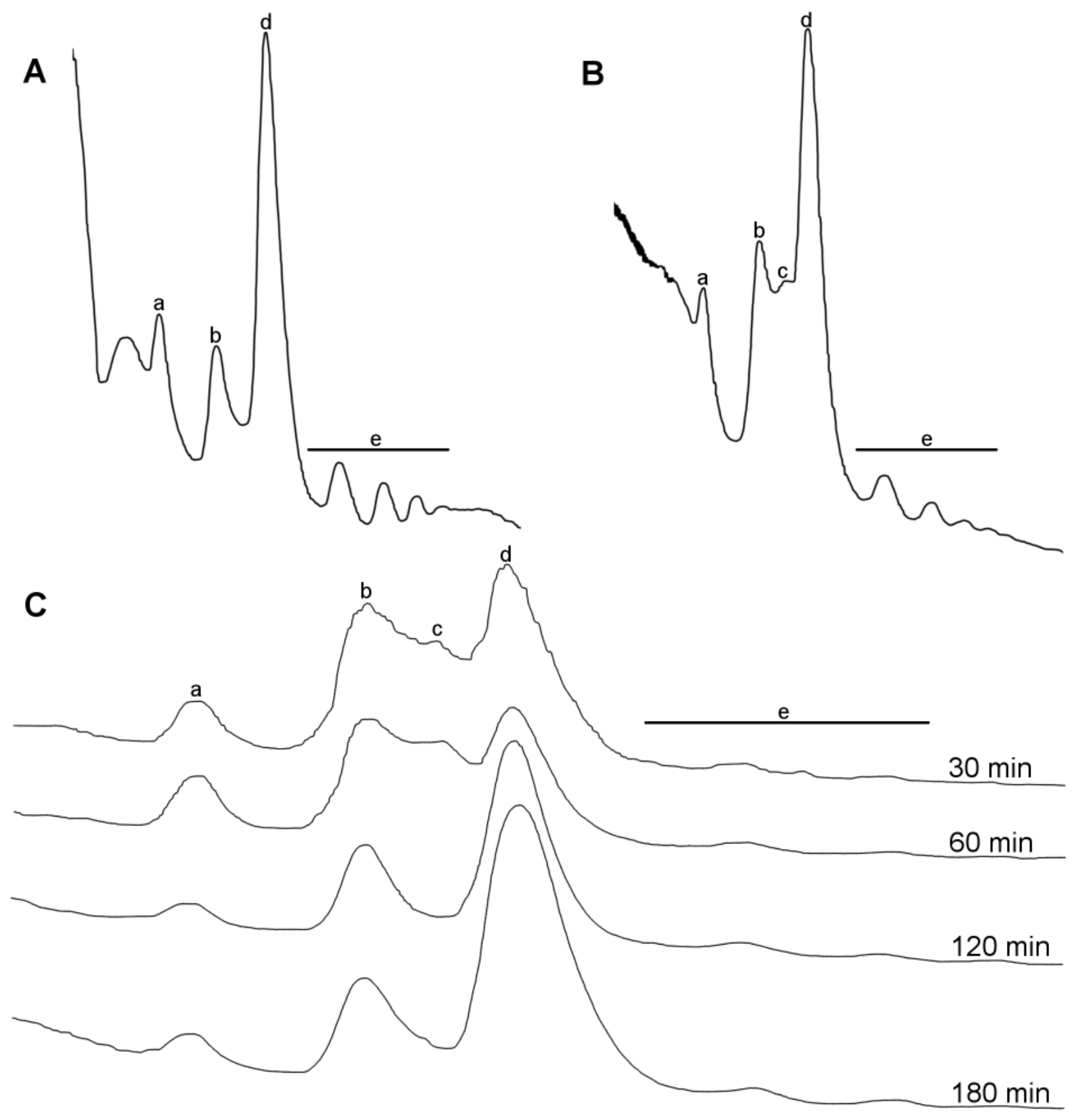

674 Figure 1. Oxidative stress induces the appearance of an undescribed peak between $67550 \mathrm{~S}$ and $70 \mathrm{~S}$. Sedimentation profiles of E. coli extracts separated in 10 to $40 \%$ sucrose 676 gradients and detected at $254 \mathrm{~nm}$. A) Samples collected from cultures at control conditions 677 and B) 30 min oxidative stress (Paraquat $250 \mu \mathrm{M}$ ). C) Similar experiments performed after 30 , 67860,120 and 180 min of oxidative stress induced by $250 \mu \mathrm{M}$ Paraquat. Lowercase letters 679 indicate $30 \mathrm{~S}(\mathrm{a}), 50 \mathrm{~S}$ (b), new peak (c), 70S (d) and polysomes (e). 
A
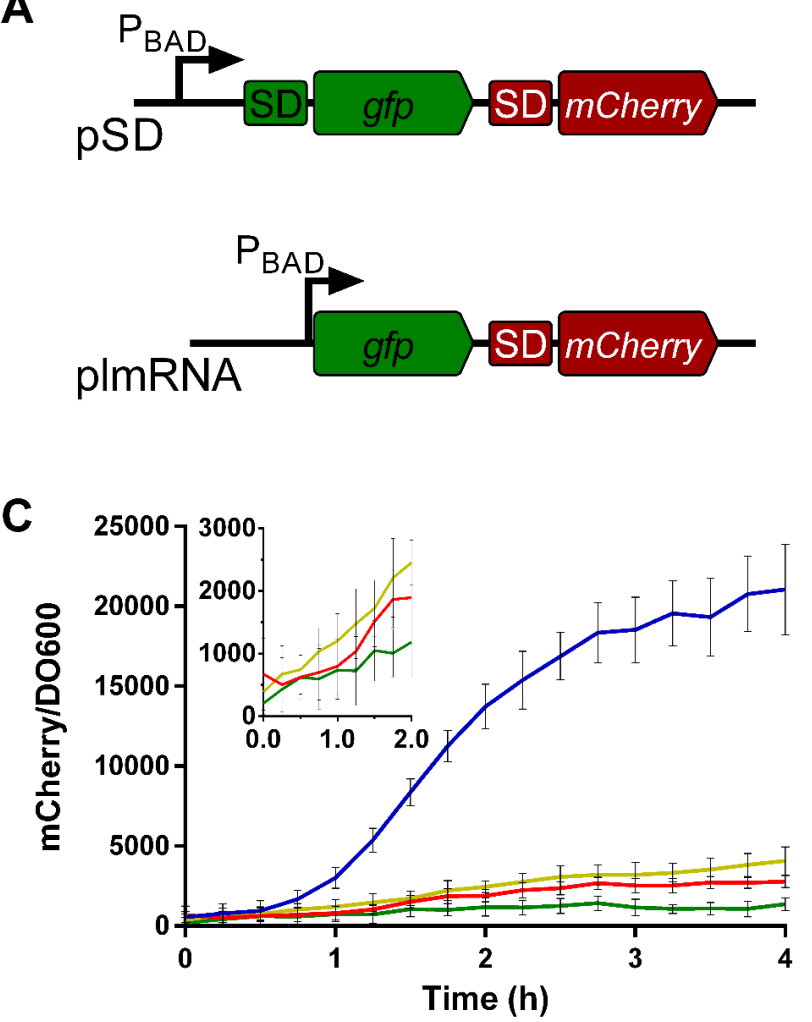

E

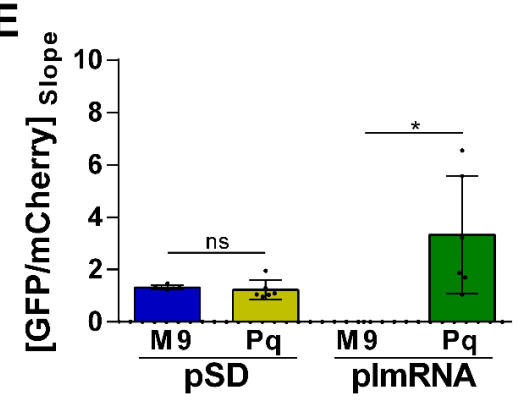

B

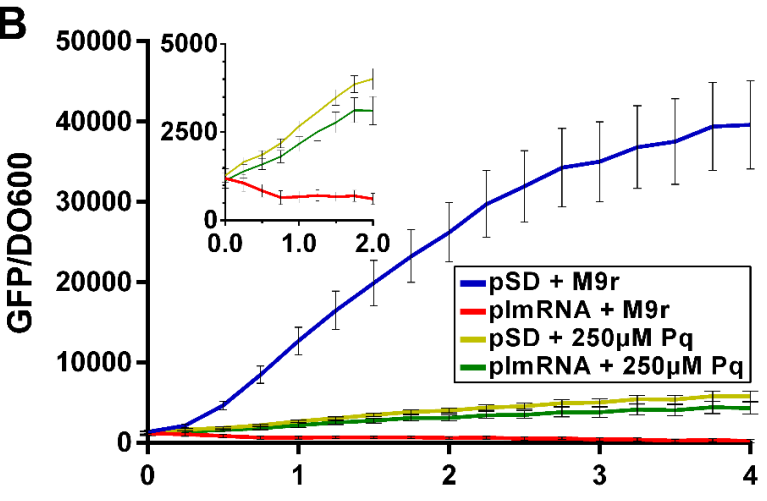

D

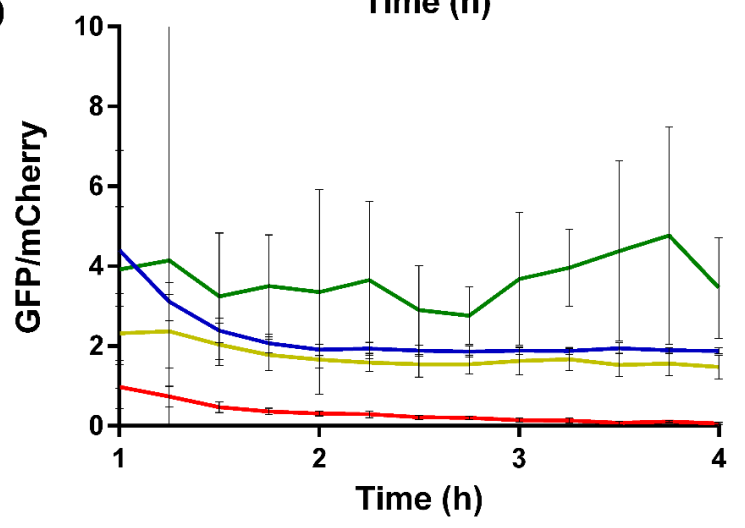

G
680

\section{1}

682

\section{3}

684

685

686

687

688

689

690

691
F

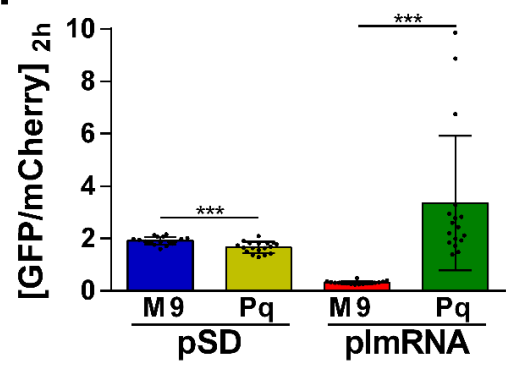

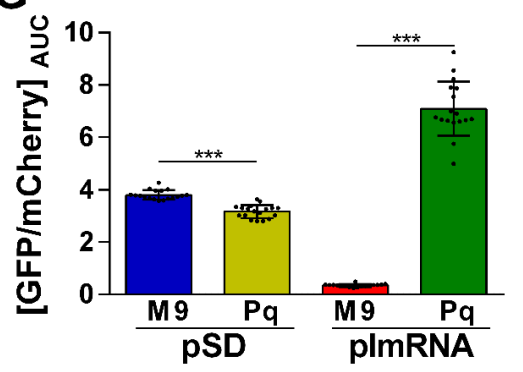

Figure 2. ImRNA is translated more efficiently under oxidative stress. A) Scheme of leader (pSD) or leaderless (plmRNA) reporters used in B, C, D, E, F and G. The biscistronic reporter system codes superfolder GFP ( $g f p$ ) and mCherry (mCherry), downstream of the $P_{\text {BAD }}$ promoter. B) and C) Kinetics of fluorescence normalized by $\mathrm{OD}_{600}$ for GFP (B) and mCherry (C). D) Kinetic of the ratio of fluorescence intensities between GFP and mCherry (GFP/mCherry) for pSD and plmRNA in control (M9) and oxidative stress (250 $\mu \mathrm{M}$ Paraquat) conditions. E) Ratio between the slopes of the linear segment of the GFP/DO600 (0,5-2,0h) and mCherry/DO600 (1-2h) kinetics, under control and stress conditions. F) GFP/mCherry ratio at $2 \mathrm{~h}$ after transcription induction, under control and stress conditions. G) Area under the curve (AUC) of the graph in figure 2B between 2 and $4 \mathrm{~h}$ after transcription induction, under control and stress condition. Statistical analyses: unpaired two tailed $t$ test with Welch's correction. ${ }^{* \star * *} p<0,0001,{ }^{* *} p<0,001,{ }^{*} p<0,05$. 

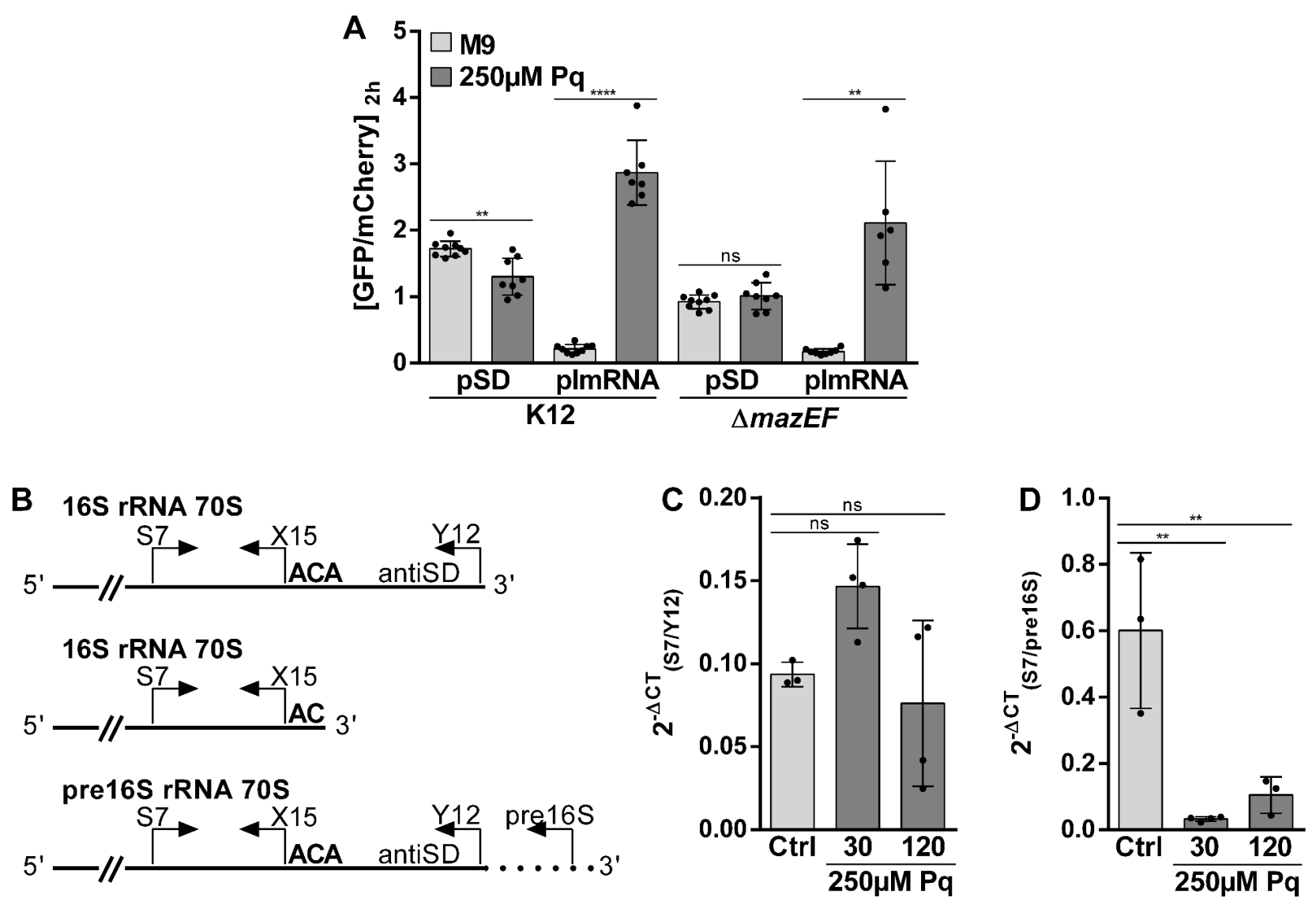

692 Figure 3. MazF and the cleavage of 16S 3' extreme are not required to induce translation under oxidative stress. A) GFP/mCherry fluorescence ratio for wild-type and $\triangle$ mazEF::FRT $(\triangle m a z E F)$ E. coli strains carrying pSD and plmRNA constructions at $2 \mathrm{~h}$ after transcription induction in control and stress condition. B) PCR strategy used to analyse the integrity of 16S rRNA 3' extreme. C) and D) Integrity analysis of 16S rRNA (C) and pre 16S rRNA (D) from wild-type E. coli under control condition and 30 or 120 min after oxidative stress induction. Statistical analyses: unpaired two-tailed t test for A. One-way ANOVA and Dunnett's multiple comparisons test for $C$ and $D .{ }^{* * *} p<0,0001,{ }^{* *} p<0,01,{ }^{*} p<0,05$. 
A

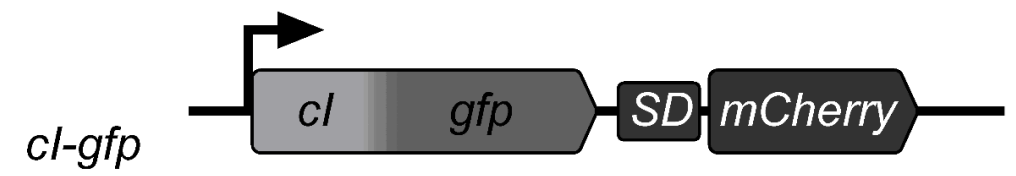

B
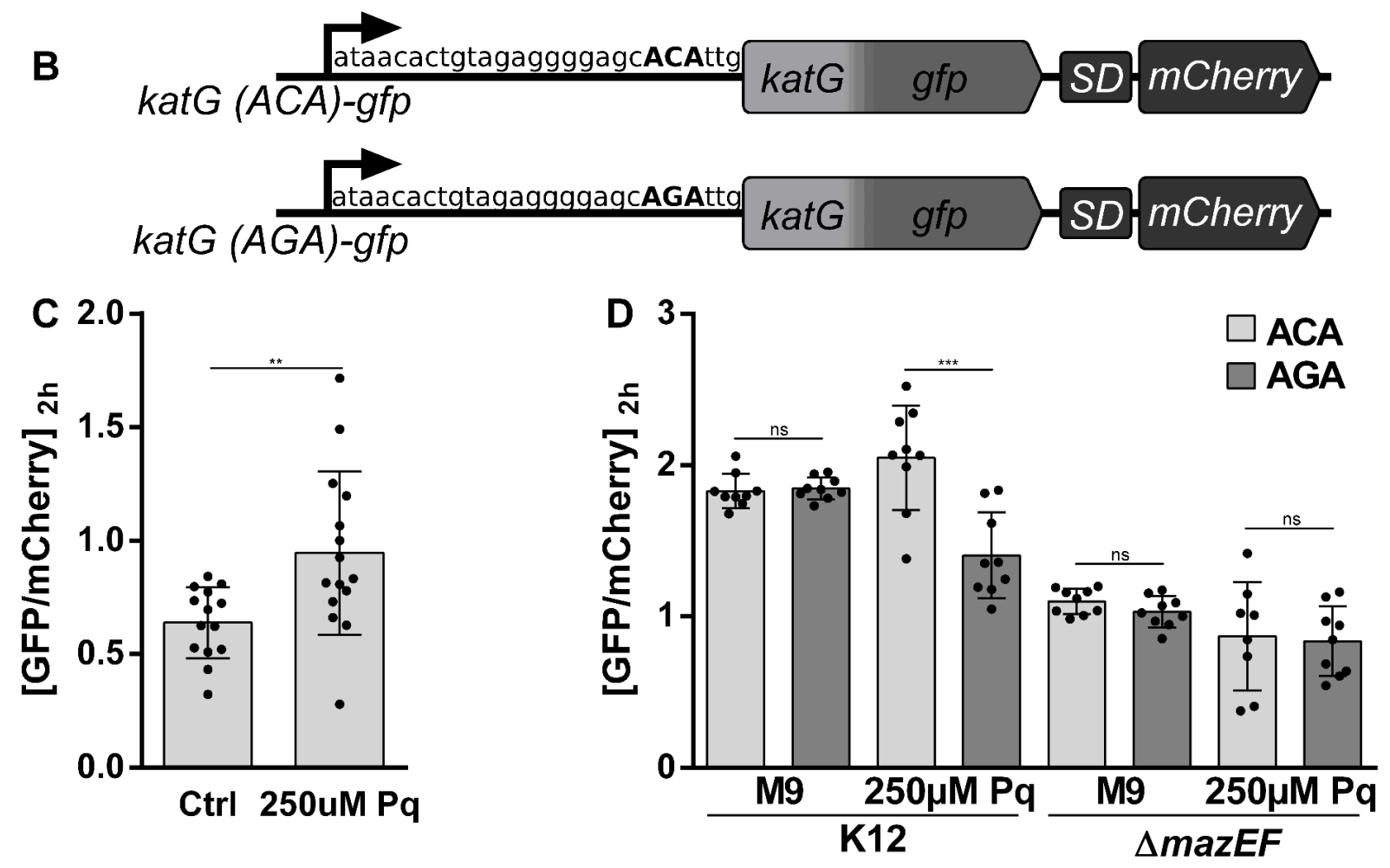

700 Figure 4. Oxidative stress increases the efficiency of translation of genes naturally

701

702

703

704

705

706

707

708

709

710

711 encoded as leaderless transcripts. A) Scheme of the translational fusion of the first 100 codons of leaderless $\mathrm{cl}$ transcript (from $\mathrm{P}_{\mathrm{RM}}$ promoter) to the gfp-mCherry reporter system. B) Scheme of the translational fusion between the 5'UTR and the first 100 codons of katG transcript to gfp-mCherry reporter system. katG(ACA)-gfp includes the wild-type 5'UTR while $\operatorname{katg}(A G A)$-gfp includes a point mutation in sequence ACA, recognized by MazF. C) GFP/mCherry fluorescence ratio of $\mathrm{cl}$-gfp fusion at $2 \mathrm{~h}$ after transcription induction, in control and stress conditions. D) GFP/mCherry fluorescence ratio of katG-gfp fusion at $2 \mathrm{~h}$ after transcription induction, in control and stress conditions in the WT and $\triangle$ mazEF strains. Statistical analyses: unpaired two-tailed t test with Welch's correction for C. Unpaired twotailed $t$ test for $D .{ }^{* \star \star} p<0,001,{ }^{* \star} p<0,01$. 

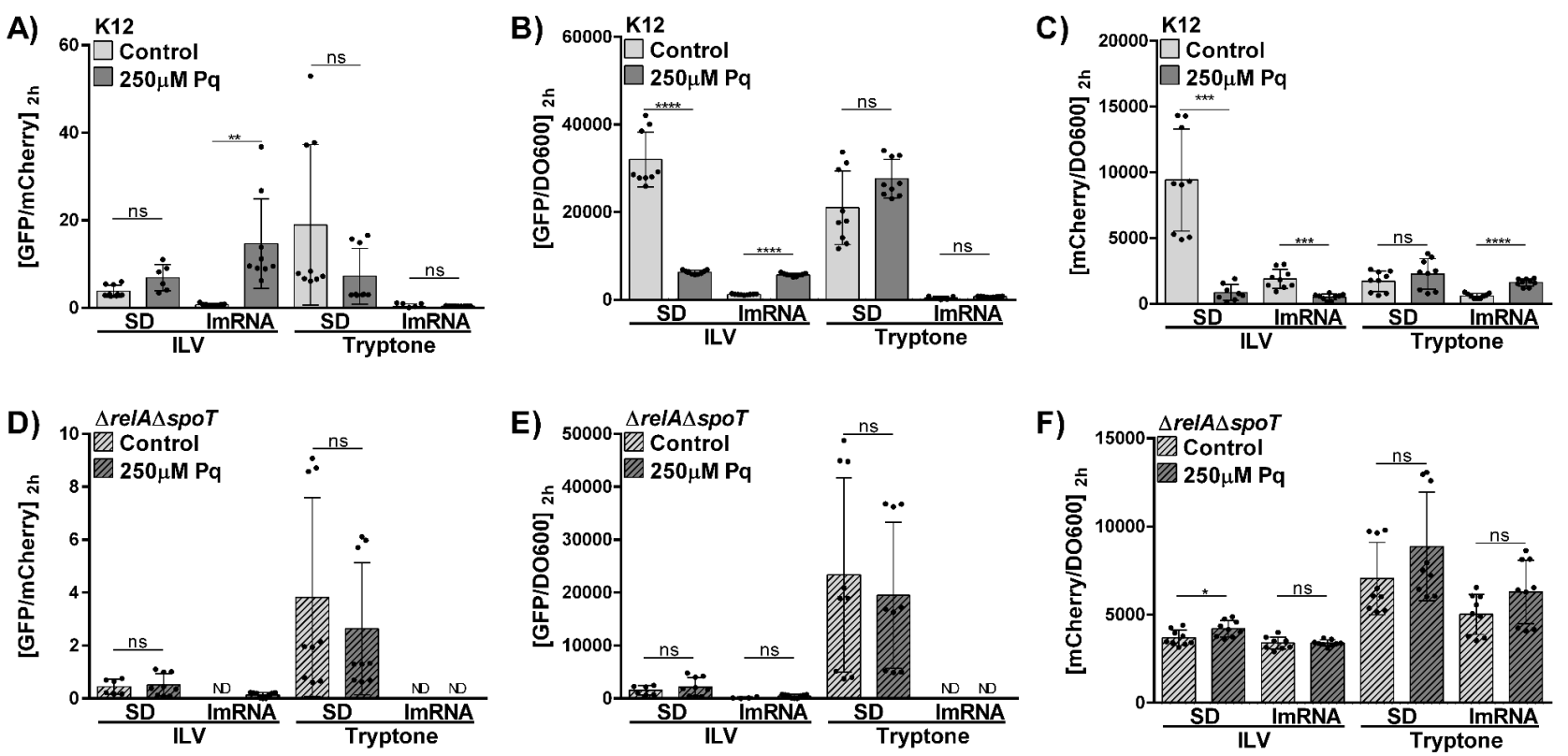

Figure 5. The induction of ImRNA translation under oxidative stress depends on the synthesis of (p)ppGpp and the availability of amino acids. GFP/mCherry, GFP/DO600 and mCherry/DO600 ratios for WT (K12; A, B and C) and $\Delta r e l A:: F R T \Delta s p o T:: F R T$ ( $\triangle$ relA $\Delta s p o T$; D, E and F) E. coli strains carrying the pSD and plmRNA constructs, at $2 \mathrm{~h}$ after transcription induction in control (light gray) and oxidative stress conditions (dark gray). The M9 culture medium was supplemented with low concentrations of branched amino acids (ILV $50 \mu \mathrm{g} / \mathrm{mL}$ each) or high concentration of all amino acids (Tryptone $0.5 \%$ ). Note that experiments in this figure were performed with three independent clones of the $\triangle$ relA $\triangle s p o T$ strain, explaining in part the higher dispersion of data. ND= Not detected. Statistical analyses: unpaired two tailed $t$ test with Welch's correction. ${ }^{* \star \star \star} p<0,0001,{ }^{* \star \star} p<0,001,{ }^{\star \star} p<0,01$, ${ }^{*} p<0,05$. 